\title{
„Leihmutterschaft ist mit der Menschenwürde nicht vereinbar"
}

\author{
Anmerkungen zum Statement der Bundesregierung. \\ Medizinische und psychologische Überlegungen
}

Zusammenfassung: Leihmutterschaft als eine Form der Kinderwunschbehandlung und schließlich als Mutterschaft wird medizinisch, ethisch und psychosozial sehr kontrovers diskutiert. In Deutschland ist die Leihmutterschaft aufgrund des Embryonenschutzgesetzes verboten. Frauen aus Deutschland gehen zu einer Leihmutterschaftsbehandlung in Länder, die dieses entweder grundsätzlich erlauben oder eine Behandlung nur aus „altruistischen Gründen“ akzeptieren.

Die vorhandene medizinische Literatur zu Schwangerschaft und Geburt bei Leihmutterschaft zeigt keine wesentlichen Besonderheiten im Vergleich zu Schwangerschaften nach anderen Formen der Sterilitätstherapie. Auch die psychologischen Nachuntersuchungen $\mathrm{zu}$ den Kindern, zu den auftraggebenden Eltern, aber auch zu den Leihmüttern sehen keine wesentlichen negativen Befunde. In der ethischen Diskussion steht vor allem die Frage im Vordergrund, wie es $\mathrm{zu}$ bewerten ist, wenn eine Schwangerschaft gegen eine finanzielle Kompensation ausgetragen wird, wobei die Belastung für die Leihmutter für die gesamte Schwangerschaftsdauer von 9 Monaten ansteht. Auch die „Übergabe des Kindes“ an eine fremde Frau wird kontrovers diskutiert bezüglich der Frage, ob die Unterbrechung der Mutter-Kind-Beziehung durch die Geburt langfristige negative Auswirkungen für die Leihmutter und das Kind haben kann. Die reproduktionsmedizinischen Fachgesellschaften und ihre Ethikkommissionen sehen hierzu keine grundlegenden Probleme.

Die Nationale Akademie der Wissenschaft (Leopoldina) hat sich in einer Stellungnahme 2019 mit dem Thema befasst und notwendige Veränderungen sowohl bei Beibehaltung des Verbots der Leihmutterschaft als auch bei einer gesetzlichen Erlaubnis formuliert.

\section{Einleitung}

Bevor die medizinischen, ethischen und psychosozialen Fragen der Leihmutterschaft erörtert werden, sollen einige (grundlegende) Vorbemerkungen erfolgen. 


\section{Statement der Bundesregierung}

Jede Bundesregierung hält in ihrem Regierungsprogramm zu Beginn der Amtszeit fest, welche politischen Probleme sie angehen will.

Zum Beginn der 18. Legislaturperiode (2013-2018) hielt die damalige Bundesregierung im Kapitel zu Gesetzesplänen im Bereich der Reproduktionsmedizin fest, dass es keine Veränderungen im Embryonenschutzgesetz geben soll.

Es findet sich dabei ein eindeutiges Statement: „Die Leihmutterschaft lehnen wir ab, da diese mit der Würde des Menschen unvereinbar ist“. (Koalitionsvertrag 2013). Seit Bestehen des Embryonenschutzgesetz ist neben der Eizellspende auch die Leihmutterschaft nach Paragraph 1 eindeutig verboten (Embryonenschutzgesetz 1990).

Der Hinweis auf die Menschenwürde (§ 1 des Grundgesetzes) ist grundsätzlich ein gravierendes Argument. Es sollte jedoch hinterfragt werden, wessen Menschenwürde gemeint ist: die der austragenden Mutter, der auftraggebenden Mutter, des auftraggebenden Vaters oder des Kindes oder aller Beteiligten.

Wenngleich dies im Regierungsprogramm nicht näher dargestellt wird, so kann es doch Aufgabe der Mediziner, Ethiker und Sozialwissenschaftler sein, diese Aussage zu hinterfragen.

\section{Kinderwunschbehandlung als Krankenbehandlung}

Noch grundsätzlicher muss aber die Frage gestellt werden, wie eine Kinderwunschbehandlung - entweder mit eigenen Spermien und Eizellen oder auch mit fremden Gameten sowie bei Leihmutterschaft - im medizinischen und juristischen Sinne zu verorten ist. Ist sie vergleichbar mit einer Krankenbehandlung im Sinne der medizinischen Behandlung, z. B. des Bluthochdrucks, einer Zuckererkrankung, einer operativen Therapie von Tumoren, die allgemein auch über das Sozialgesetzbuch V im $\S 27$ als „Krankenbehandlung“ definiert sind - mit der Konsequenz, dass die gesetzliche Krankenversicherung diese auch komplett bezahlt?

Es lässt sich darüber streiten, ob Sterilität eine Krankheit ist. In jedem Fall leidet im psychischen Sinne das Paar an ungewollter Kinderlosigkeit, wenn es sich in eine medizinische Diagnostik und Therapie begibt. Man kann diese Form der Therapie aber durchaus auch als eine „wunscherfüllende Medizin“ begreifen, denn im engeren Sinne sollen Wünsche (hier Kinderwunsch) behandelt werden und nicht unbedingt eine klar definierbare Krankheit. Der Gesetzgeber hat dies dadurch gelöst, dass er im Sozialgesetzbuch V einen isolierten § 27a (Künstliche 
Befruchtung) aufgenommen hat, in dem er regelt, dass die gesetzlich versicherten Paare unter bestimmten Voraussetzungen (Ehestatus und Altersgrenzen) Anspruch auf (partielle) Bezahlung einer Kinderwunschbehandlung haben. Dieses gilt insbesondere für Maßnahmen der künstlichen Befruchtung (In-vitro-Fertilisation) und intrazytoplamatischen Spermieninjektion (ICSI), bei der eine einzelne Spermie in die Eizelle injiziert wird.

\section{Sind Paare mit Kindern glücklicher?}

Bei der Frage, ob eine Kinderwunschbehandlung eher die Behandlung eines Leidensdrucks oder einer Krankheit ist, sollte zugleich auch bedacht werden, wie es den Paaren nach erfülltem Kinderwunsch (Geburt eines Kindes) im Vergleich zu Paaren geht, die „erfolglos“ geblieben sind. Hierüber gibt die AWMF-Leitlinie „Psychosomatisch orientierte Diagnostik und Therapie von Fertilitätsstörungen“ (2020) Auskunft und hält im Statement fest:

„Die langfristige Entwicklung der psychosozialen Situation von Paaren nach erfolgloser reproduktionsmedizinischer Behandlung zeigt, dass der unerfüllte Kinderwunsch oft noch eine große Rolle im Leben der Paare spielt. Infertilität wird von vielen Betroffenen als belastende Episode im Leben empfunden. Die meisten Paare bewältigen langfristig die Situation und sind in ihrem psychischen Wohlbefinden später nicht mehr beeinträchtigt.

In der Lebensqualität und der Lebenssituation zwischen Kinderlosen und Personen mit Kindern nach Kinderwunschbehandlung bestehen langfristig nur geringe Unterschiede. Ungewollte Kinderlosigkeit bleibt jedoch für einige Betroffene ein Lebensereignis, welches immer wieder Gefühle des Bedauerns auslösen (z.B. in Lebensphasen wie Klimakterium oder Übergang Gleichaltriger in die Großelternschaft) und erneute Adaptationsleistungen erfordern kann.

Die ungewollte Kinderlosigkeit wird zu einer andauernden Belastung, wenn die Fähigkeiten zur Entwicklung neuer Lebensperspektiven eingeschränkt sind. Diese Fähigkeiten werden von der psychischen Prädisposition sowie dem Verlauf der Infertilitätskrise, den Kinderwunschmotiven und der Kinderwunschintensität, der Partnerschaftszufriedenheit und der Ursachenzuschreibung beeinflusst. Als ungünstiger Prognosefaktor hat sich eine starke soziale Isolierung erwiesen.“

Soweit zur Lebensqualität von Menschen mit Kinderwunsch.

Es stellt sich weiter die Frage, wie im weiteren Verlauf des Lebens generell die Lebensqualität der Paare mit oder ohne Kinder einzuschätzen ist - unabhängig von der Frage des Kinderwunsches. Eine soziologische Studie hält zu dieser Frage fest (Deaton/Stone 2014): 
„Viele Leute denken, dass ihre Kinder ihr Leben verbessern. Aber viele Studien zeigen, dass Menschen ohne Kinder ihr Leben besser bewerten als Menschen mit Kindern. Allerdings gibt es einen kleinen Effekt, dass Menschen mit Kindern über günstigere Lebensbedingungen verfügen in Bezug auf ein besseres Leben. Eltern erfahren täglich mehr Freude, aber auch Stress als „Nicht-Eltern“. Dieses zu interpretieren ist schwierig, da es um die grundsätzliche Frage einer Lebensentscheidung geht. Wenn es die grundsätzliche Entscheidung für oder gegen Elternschaft gab, dann gibt es kaum Gründe, dass die eine Lebensform besser ist als die andere Lebensform.“

\section{Möglichkeiten und Erfolge der Kinderwunschbehandlung}

Wenngleich also die Einordnung einer ungewollten Kinderlosigkeit und der Sterilitätsmedizin im Gesundheitssystem schwierig ist, so lässt sich aber festhalten, dass mittlerweile die Diagnostik von Fertilitätsstörungen und insbesondere die Behandlung recht erfolgreich sind.

Standardverfahren sind eine Hormonkorrektur, wenn Hormonstörungen insbesondere bei der Frau vorliegen (zum Beispiel zu viel männliche Hormone, Schilddrüsenstörung).

Wenn Störungen der Eizellreifung vorhanden sind, so lässt sich dieses mit Medikamenten zur Hormonstimulation ebenfalls gut therapieren.

Seit der Geburt des ersten IVF-Kindes 1978 hat sich die In-vitro-Fertilisation (später im Zusammenhang mit der intrazytoplasmatischen Spermieninjektion (ICSI)) zu einem Standardverfahren entwickelt.

Wesentlich für die Paare sind die Erfolge im Sinne einer Geburtenrate pro Eizellentnahme, wenn nach hormoneller Vorbehandlung unter Narkose Eizellen entnommen werden, um sie mit dem Samen des Partners/Mannes oder fremden Samen zu befruchten. Mittlerweile liegen auch in Deutschland zufriedenstellende Zahlen vor: Pro Eizellpunktion kann die Patientin mit einer Geburtenrate von 20$22 \%$ rechnen. (Die Schwangerschaftsrate liegt eindeutig höher: Hier sind aber die Fehlgeburten abzuziehen.) (DIR 2019).

\section{Nochmal zu Verboten}

Viele Formen der Kinderwunschbehandlung sind in Deutschland möglich.

In Deutschland ist allerdings neben der Leihmutterschaft weiterhin die Eizellspende vom Verbot betroffen. Deswegen gehen von Deutschland aus mehrere 1000 Frauen pro Jahr ins Ausland, um dort die entsprechende Behandlung 
durchführen zu lassen, wenn sie selbst keine oder nicht genügend Eizellen haben, um eine Schwangerschaft herbeizuführen. Von diesem Problem sind 2\% aller Frauen bis zum 40. Lebensjahr betroffen.

Kommen wir auf den Ausgangspunkt zurück: Es steht die Frage im Vordergrund, ob sich das Statement der Bundesregierung, dass die Leihmutterschaft „nicht mit der Menschenwürde vereinbar ist“, im Licht vorhandener medizinischer Daten, aber auch einer ethischen und psychosozialen Debatte aufrecht erhalten lässt. Dieses soll nachfolgend erörtert werden.

Im Hintergrund bleibt aber das Statement, dass Kinderwunschbehandlungen (incl. Leihmutterschaft) als „wunscherfüllende“ Medizin anzusehen sind, so dass die Bedingungen dieser Medizin sowie die Eingebundenheit von allen Beteiligten näher zu beleuchten sind.

\section{Allgemeine Grundlagen}

Leihmutterschaft (Surrogacy) ist in einigen Ländern eine anerkannte Behandlungsmethode bei nicht erfüllbarem Kinderwunsch, bei der eine Frau eine Schwangerschaft für eine andere (auftraggebende) Frau austrägt.

In Deutschland ist die Leihmutterschaft verboten. Im Folgenden sollen Definition, Rechtslage, Indikation, medizinische und psychologische Aspekte sowie ethische Fragen erörtert werden.

\subsection{Definition}

Eine Leihmutter ist eine Frau, die für eine auftraggebende Frau oder ein Paar ein Kind austrägt.

Als Formen der Leihmutterschaft sind möglich (Tschudin/Griesinger 2012):

- Vollumfängliche Leihmutterschaft

Hierbei ist keine genetische Verwandtschaft der Leihmutter mit dem Kind vorhanden. Die Gameten können von beiden auftraggebenden Eltern, von einem Elternteil oder von keinem Elternteil stammen (Embryonenspende). Die Schwangerschaft entsteht durch Embryotransfer.

- Teilweise Leihmutterschaft

Es besteht eine genetische Verwandtschaft der Leihmutter mit dem Kind. Z. B. könnte die Schwangerschaft durch Insemination bei der Leihmutter entstanden sein. 
Eine Leihmutterschaft ist auch innerhalb der Familie möglich (z.B. bei der Schwester oder von Mutter zu Tochter oder Tochter zu Mutter). Hier bestehen sehr hohe Anforderungen an Information, Aufklärung und Beratung (ESHRE Task Force on Ethics and Law 2011).

\subsection{Rechtslage}

Die Leihmutterschaft ist in Deutschland nach dem Embryonenschutzgesetz verboten. Grundsätzlich regelt § 1591 Bürgerliches Gesetzbuch (BGB) in Deutschland, dass die Mutter eines Kindes die Frau ist, die es geboren hat. Innerhalb Europas ist die Leihmutterschaft juristisch erlaubt u.a. in Großbritannien, Belgien, Griechenland, Spanien und den Niederlanden; außerhalb Europas ist sie u.a. in Israel, Australien, Russland sowie in den meisten Staaten der USA möglich.

Paare aus Deutschland gehen daher für eine mögliche Leihmutterschaft vor allem in diese Länder. Die Paare selbst machen sich nicht strafbar. Da die rechtliche Handhabung zur Leihmutterschaft innerhalb Europas und weltweit unterschiedlich ist, müssen die auftraggebenden Eltern und die Leihmütter die gesetzlichen Rahmenbedingungen des betreffenden Landes (insbesondere zur Adoption) berücksichtigen (Tschudin/Griesinger 2012, Kentenich 2018).

\subsection{Indikationen}

Die ESHRE Task Force on Ethics and Law (2005) sieht folgende mögliche Indikationen:

- fehlender Uterus (z. B. Mayer-Rokitansky-Küster-Hauser-Syndrom, Z.n. Hysterektomie (Gebärmutterentfernung) z.B. wegen Krebserkrankung)

- Uterus ohne funktionsfähiges Endometrium (z. B. nicht behandelbares Asherman-Syndrom mit Verlust der Gebärmutterschleimhaut)

Kontraindikationen:

- alle Kontraindikationen gegen eine Schwangerschaft aufgrund schwerwiegender Grunderkrankung der Leihmutter

In einigen Ländern werden auch Leihmutterschaften aus sozialen Gründen durchgeführt, ohne dass eine medizinische Indikation besteht. Dieses soll hier nicht erörtert werden.

Die Gameten können von den auftraggebenden Eltern, von der Leihmutter oder von Dritten stammen. 


\subsection{Grundsätzliche Überlegungen}

Bei der Leihmutterschaft sind unterschiedliche Rechte und Gefahren für alle beteiligten Personen wie Leihmutter, Wunscheltern und Kind abzuwägen. Es besteht ein hohes Missbrauchspotential aufgrund der möglichen erheblichen sozialen Ungleichgewichte zwischen den auftraggebenden Eltern und den Leihmüttern. In einigen Ländern dient das Entgelt für die Leihmutterschaft der Finanzierung des Unterhalts einer ganzen Familie. Es ist daher ein erheblicher Unterschied, ob die Motivation aus eher altruistischen oder eher kommerziellen Motiven erfolgt. Wenngleich in der Befragung der Leihmütter oft auch altruistische Motive angegeben werden, so ist es wenig wahrscheinlich, dass das Austragen der Schwangerschaft für fremde Paare über neun Monate aus ausschließlich altruistischen Motiven erfolgt, ohne dass finanzielle Aspekte eine Rolle spielen.

In Ländern wie Großbritannien ist eine Leihmutterschaft nur möglich mit Beschränkung der finanziellen Kompensation. Die Kompensation liegt bei 10.000 bis 13.000 Pfund (HFEA 2016).

Es ist zu bedenken, dass die Leihmutter über mehrere Monate hinweg ihren Körper zum Austragen der Schwangerschaft zur Verfügung stellt. Sie kann durchaus in dieser Zeit ihre Meinung zum Austragen der Schwangerschaft ändern.

Ein Verbot der Leihmutterschaft wäre allerdings ein deutlicher Eingriff in das grundsätzlich geschützte Recht auf Fortpflanzungsfreiheit. Zugleich birgt das Verbot der Leihmutterschaft in Deutschland für viele Paare (sowohl heterosexuelle als auch homosexuelle Paare), die ins Ausland reisen, die Gefahr, dass das geborene Kind keine eindeutige rechtliche Zuordnung $\mathrm{zu}$ einem oder beiden (auftraggebenden) Elternteilen erhält.

\subsection{Medizinische Aspekte zur Schwangerschaft}

Für eine Leihmutter bestehen die üblichen mütterlichen Risiken einer Schwangerschaft (z.B. Entwicklung von Schwangerschaftshochdruck/ Präeklampsie) oder auch für die Schwangerschaft an sich (z.B. Fehlgeburt oder Eileiterschwangerschaft sowie Mehrlingsschwangerschaften) (Söderström-Anttila et al. 2016).

Grundsätzlich sollte nur ein Embryo übertragen werden, um Mehrlingsschwangerschaften zu vermeiden, da Mehrlingsschwangerschaften häufig zu Frühgeburten führen mit langfristigen negativen Folgen für die physische und psychische Gesundheit der Kinder.

Wenn die Eizellen der Leihmutter verwendet werden, so sollte dies aufgrund des Alters eine hinreichende Aussicht auf Erfolg bieten: Je älter eine Frau ist, von 
der die Eizellen stammen, umso geringer ist der Erfolg einer Geburt, da die Mehrzahl der Eizellen keinen korrekten („euploiden“) Chromosomensatz haben.

Die Raten an Mehrlingsgeburten und Fehlgeburt scheinen mit anderen Schwangerschaften nach künstlicher Befruchtung vergleichbar zu sein (Söderström-Anttila et al. 2016). Neben den medizinischen Aspekten stehen psychologische Aspekte im Vordergrund, da die Leihmutter selbst entscheidet, ob und wie häufig sie pränatale Untersuchungen durchführt, ob sie sich entsprechend „schonend“ verhält (z. B. kein Rauchen, kein Alkohol) und ob sie den üblichen Empfehlungen für den Schwangerschaftsverlauf und Geburtsmodus folgt (Vorsorgeuntersuchungen, Spontangeburt oder Sectio).

Diese Entscheidungen sind im Lichte ihrer eigenen Autonomie $\mathrm{zu}$ sehen. Letztlich kann die Leihmutter bei Vorliegen entsprechender juristischer Voraussetzungen sogar einen Schwangerschaftsabbruch durchführen lassen.

Die Zahl der Embryotransfere bei Leihmutterschaftsbehandlung stieg in den USA von 1957 (2\% aller IVF-Behandlungen) im Jahre 2007 auf 5521 im Jahre 2014 (4\% aller IVF-Behandlungen) (CDC 2016).

In Großbritannien lag die Zahl bei 0,4\% aller IVF-Behandlungen also deutlich niedriger (HFEA 2017), was mit der strengeren Regulierung zusammenhängen dürfte. Im Jahre 2016 wurden 232 Behandlungszyklen mit Leihmutterschaft in Großbritannien durchgeführt (HFEA 2016).

Es wird in einer Übersicht über klinische Schwangerschaftsraten von 19-33\% pro Embryotransfer berichtet. 30-70\% der auftraggebenden Paare wurden schließlich Eltern (Söderström-Antilla et al. 2016). Auf Grundlage dieser Daten handelt es sich um ein Verfahren, welches im Vergleich zu anderen Verfahren der künstlichen Befruchtung als relativ erfolgreich anzusehen ist. Schwangerschaftsund Geburtsraten entsprechen dem „normalen“ IVF-Verfahren.

Daten zur weltweiten Übersicht der Reproduktionsmedizin (ICMART 2020) zeigen keine auswertbaren Zyklen zur Leihmutterschaft.

\subsection{Psychologische Erwägungen}

Die psychologischen Untersuchungen haben die psychosozialen Auswirkungen auf die betroffenen Parteien, insbesondere auf die auftraggebenden Eltern, das Kind sowie die Leihmutter (Tschudin/Griesinger 2012) zum Inhalt.

Van der Akker (2007) beschäftigte sich in einer Längsschnittuntersuchung mit dem Persönlichkeitsmerkmalen von Frauen, die als Leihmütter für eine andere Frau ein Kind austragen wollen und Frauen, die eine eigene Schwangerschaft austragen wollen. Die Studie startete vor der Schwangerschaft und erfasste auch den Zeitraum bis 6 Monate postpartal. Persönlichkeitsmerkmale und Ängstlich- 
keit waren nicht wesentlich unterschiedlich. Frauen mit „eigener Schwangerschaft“ (keine Leihmutterschaft) waren am Ende der Schwangerschaft etwas ängstlicher, wenn der Foet im Ultraschall sichtbar wurde. Obwohl Leihmütter weniger ängstlich waren, so sieht die Autorin die Notwendigkeit der psychozialen Beratung und Unterstützung von Leihmüttern.

Die Arbeitsgruppe um Golombok führte Longitudinaluntersuchungen durch bezüglich der Kinder, die nach Leihmutterschaft geboren wurden, sowie der auftraggebenden Eltern. Diese wurden verglichen mit Familien nach Eizellspende und nach Spontankonzeption. Die Autoren fanden keine Hinweise, dass sich die Leihmutterschaft oder der fehlende genetische Hintergrund negativ auf die ElternKind-Beziehung, auf das psychische Wohlergehen der Mütter, Väter und Kinder auswirken (Söderström-Anttila et al. 2016, Golombok et al. 2004, 2006 a, 2006 b, 2011, Jadva et al. 2012).

Etwa ein Drittel der Leihmütter berichtet über leichte und mäßige Schwierigkeiten bei der Übergabe des Kindes. Dieser Anteil war signifikant höher, wenn es sich bei den Leihmüttern um Frauen handelte, die den auftraggebenden Eltern schon vorher bekannt waren (Tschudin/Griesinger 2012). Knapp 10\% der Leihmütter suchten während oder nach der Schwangerschaft wegen psychologischer Probleme den Hausarzt oder einen Psychologen auf (Tschudin/Griesinger 2012; Jadva et al. 2003). Der Kontakt der Kinder zu den Leihmüttern wird als harmonisch beschrieben. Im Alter von 10 Jahren waren 90 Prozent der Kinder über die Art ihrer Konzeption (Leihmutterschaft) informiert. Dieses wirkte sich im Wesentlichen positiv auf die Beziehung zwischen den Kindern und Leihmüttern aus (Jadva et al. 2012).

Insgesamt ist die Datenlage zu Nachuntersuchungen bei Leihmutterschaft begrenzt. Die psychologischen Untersuchungen lassen aber den Schluss zu, dass die Eltern-Kinder-Beziehung und die Entwicklung der Kinder unauffällig zu sein scheinen. Problematisch kann ein kontrollierendes Verhalten der auftraggebenden Mutter/Eltern gegenüber der Leihmutter in der Schwangerschaft sein. Auch die Übergabe des Kindes von der Leihmutter auf die auftraggebenden Eltern kann psychische Schwierigkeiten beinhalten. Endgültige Aussagen sind allerdings wegen der begrenzten Anzahl der Untersuchungen nicht möglich (SöderströmAntilla et al. 2016).

\subsection{Ethische Aspekte}

Grundlage der Behandlung und Einwilligung ist das Prinzip der Autonomie, da die Freiwilligkeit als oberstes Prinzip anzuerkennen ist. Die ESHRE Task Force on Ethics and Law (2005) schätzt die bestehenden moralischen Einwände gegenüber 
einer Leihmutterschaft sowie die damit verbundenen Risiken und Komplikationen als nicht so schwerwiegend ein, dass sie ein gänzliches Verbot rechtfertigen würden (Tschudin/Griesinger 2012; ESHRE Task Force on Ethics and Law 2005). Ähnlich äußert sich das Ethic Committee der Amerikanischen Gesellschaft für Reproduktionsmedizin (2013).

Bedeutsam erscheinen die Aspekte der Bezahlung.

Grundlage sollte eine Leihmutterschaft auf altruistischer Basis sein. Dennoch sind die entstehenden Kosten und Mühen mit in die Festlegung eines finanziellen Entgelts einzubeziehen. Eine Instrumentalisierung oder Ausbeutung des menschlichen Körpers der Leihmutter und ihrer Persönlichkeit muss vermieden werden.

Insofern stehen die Aspekte der Information, Aufklärung, Beratung und die Herstellung eines informed consent im Vordergrund. Hierbei ist darauf zu achten, dass genügend Zeit für alle beteiligten Parteien im Beratungsprozess vor einer endgültigen vertraglichen Fixierung bleibt.

\section{Aspekte der Beratung und Betreuung der Leihmutterschaft}

Die Daten zu medizinischen, psychosozialen und juristischen Aspekten machen deutlich, dass die Beratungserfordernisse für alle Beteiligten im Zusammenhang mit einer Leihmutterschaft sehr hoch sind. Ärzte in Deutschland sollten bei einer Erörterung einer Leihmutterschaft mögliche Alternativen wie Verzicht auf ein Kind, die Möglichkeiten der Adoption im Inland und Ausland sowie von Pflegschaften in den Beratungsprozess mit einbeziehen. Der Arzt muss darauf hinweisen, dass die Leihmutterschaft in Deutschland verboten ist und er sollte eine juristische Beratung zu den entsprechenden juristischen Voraussetzungen des betreffenden Landes empfehlen, in dem das Paar eine Leihmutterschaft möglicherweise anstrebt. Zugleich sind rechtsphilosophische Bedenken zum Status von Leihmutterschaftsverträgen gegeben (s. Beitrag von Anca Gheaus).

Sollte der Arzt eine direkte medizinische Unterstützung durchführen, so läuft er Gefahr, dass er an einer Beihilfe zur Straftat beteiligt ist, die nach dem Embryonenschutzgesetz verboten ist (Bundesärztekammer 2016).

Zugleich sollte der Arzt insbesondere eine negative Stigmatisierung der Paare vermeiden, die bei medizinischer Unmöglichkeit ein eigenes Kind zu bekommen, eine Leihmutterschaft im Ausland erwägen und diese evtl. auch durchführen.

Es mehren sich die juristischen Stimmen in Deutschland, in einem Fortpflanzungsmedizingesetz die Leihmutterschaft $\mathrm{zu}$ erlauben und $\mathrm{zu}$ regeln 
(Gassner et al. 2013), da die Verbotsgründe für die Zukunft nicht tragfähig genug erscheinen.

\section{Stellungnahme der Nationalen Akademie der Wissenschaft (Leopoldina)}

Die Leopoldina hat sich als Nationale Akademie der Wissenschaften mit dem Stand der Reproduktionsmedizin in einer ausführlichen Stellungnahme „Fortpflanzungsmedizin in Deutschland - für eine zeitgemäße Gesetzgebung“ (2019) beschäftigt. Auf Grundlage der Erfahrungen mit dem Embryonenschutzgesetz und der raschen Weiterentwicklung von Wissenschaft und Medizin kommt sie zu der Einschätzung, dass bestimmte Festlegungen (keine Auswahlmöglichkeit von entstandenen Embryonen) zu der Situation führen, dass in Deutschland zu viele Embryonen in die Gebärmutter transferiert werden mit einer sehr hohen Rate an Zwillingsgeburten - mit allen negativen Konsequenzen für Mütter und Kinder. Auch das Verbot der Eizellspende wird hinterfragt und die Empfehlung ausgesprochen, dieses Verbot aufzuheben.

Die Leopoldina beschäftigt sich auch mit der Leihmutterschaft und sieht in der Aufarbeitung der Probleme durchaus Gründe, die für eine Aufrechterhaltung des Verbots sprechen, aber auch Gründe, die für eine Freigabe bzw. Regulierung der Leihmutterschaft sprechen.

Es erfolgt keine Festlegung einer Empfehlung, aber es gibt zwei verschiedene Handlungsoptionen.

In jedem Fall sollte „im Sinne des Kinderwohls [ ...] für im Ausland nach dem dortigen Recht legalerweise von einer Leihmutter geborene, jedoch in Deutschland aufwachsende Kinder, eine rechtlich sichere Zuordnung des Kindes zu den Wunscheltern ermöglicht werden, da von ihr zahlreiche Rechtsfolgen wie die elterliche Sorge, Unterhaltsansprüche und die Staatsangehörigkeit abhängen.

Eine in Deutschland angebotene und durchgeführte medizinische und psychosoziale Beratung zu den Problemen einer Leihmutterschaft sollte nicht strafbar sein.“

Es wird aber auch eine Option für eine Erlaubnis der Leihmutterschaft formuliert. Hier werden im Wesentlichen folgende Forderungen erhoben:

- kein kommerzieller Anreiz, jedoch angemessene Aufwandsentschädigung

- sorgsame Auswahl der Leihmutter nach medizinischen und psychosozialen Kriterien

- die Leihmutter sollte nach der Geburt innerhalb einer Bedenkzeit von wenigen Wochen über die Abgabe des Kindes an die Wunscheltern entscheiden 
können, bis zu diesem Zeitpunkt muss sie alle Entscheidungsrechte über sich und das Kind behalten

- umfassende medizinische und psychosoziale Vorbereitung und Begleitung bei Leihmutterschaft

- Notwendigkeit einer Begleitforschung aus medizinischer und psychosozialer Sicht

Folglich lässt die Leopoldina diese unterschiedlichen Optionen stehen und stellt sie zur Diskussion.

\section{Zusammenfassende Erwägungen}

Ebenso wie andere Formen der medizinischen Kinderwunschbehandlung ist auch die Leihmutterschaft als „wunscherfüllende“ Medizin zu bewerten.

Die Erfahrungen mit Leihmutterschaft sind begrenzt, so dass die Bewertung kontrovers diskutiert wird.

Obwohl Leihmutterschaft in Deutschland verboten ist, kann sich für Frauen ohne Gebärmutter oder ohne funktionsfähige Gebärmutterschleimhaut (Asherman-Syndrom) die Frage nach der Durchführung einer Leihmutterschaft stellen.

Der beratende Arzt sollte auf mögliche Alternativen und die juristischen Voraussetzungen hinweisen und mit der Patientin die medizinischen und psychosozialen Besonderheiten besprechen.

Der Schwangerschafts- und Geburtsverlauf nach Leihmutterschaft und auch die psychologischen Nachuntersuchungen bei allen Beteiligten zeigen im Wesentlichen unauffällige Ergebnisse - bei begrenzter Datenlage.

Ethische Gesichtspunkte, dass eine andere Frau auf altruistischer Basis oder gegen Entgelt ihren Körper zum Austragen einer Schwangerschaft zur Verfügung stellt und das Kind dann später an die auftraggebenden Eltern übergibt, bedürfen einer intensiven Erörterung der Interessen aller Beteiligten.

\section{Literatur}

AWMF Leitlinie (2020): Psychosomatisch orientierte Diagnostik und Therapie bei Fertilitätsstörungen (016-003; Stand 16.12.2019, gültig bis 15.12.2024), www.awmf.org. Bundesärztekammer (2016): Stellungnahme der zentralen Ethikkommission: „Umgang mit medizinischen Angeboten im Ausland ethische und rechtliche Fragen des „Medizintourismus“, 25.11.20216; https://www.zentrale-ethikkommission.de/ stellungnahmen/medizintourismus/ 
CDC (2016): Assisted Reproductive Technology. National Summary Report, https://www.cdc.gov/art/pdf/2016.

Deaton, Angus/Stone, Arthur A. (2014): Evaluative and hedonic wellbeing among those with and without children at home, in: Proceedings of the National Academy of Sciences of the United States of America 111/4, 1328-1333.

DIR (2019): Deutsches IVF-Register. Jahrbuch 2018, in: Journal für Reproduktionsmedizin und Endokrinologie, Sonderheft 1/2019.

Embryonenschutzgesetz (1990): Gesetz zum Schutz von Embryonen (ESchG) zuletzt geändert am 21. November 2011.

ESHRE Task Force on Ethics and Law 10 including Shenfield, Francoise/Pennings, Guido/Cohen, Jonathan /Devroey, Paul/de Wert, Guido/Tarlatzis, Basil (2005): Surrogacy, in: Human Reproduction 20/10, 2705-2707.

ESHRE Task Force on Ethics and Law including de Wert, Guido/Dondorp, Wybo/ Pennings, Guido/Shenfield, Francoise/Devroey, Paul/Tarlatzis, Basil/Barri, Pedro/Diedrich, Klaus (2011): Intra-familial medically assisted reproduction, in: Human Reproduction 26/3, 504509.

Ethics Committee of the American Society for Reproductive Medicine (2013): Consideration of the gestational carrier: a committee opinion, in: Fertility and Sterility 99/7, 1838-1841.

Gassner, Ulrich/Kersten, Jens/Krüger, Matthias/Lindner, Josef Franz/Rosenau, Henning/Schroth, Ulrich (2013): Fortpflanzungsmedizingesetz Augsburg-Münchner-Entwurf (AME-FMedG), Tübingen.

Gheaus, Anca: Die normative Bedeutung der Schwangerschaft stellt Leihmutterschaftsverträge in Frage, im vorliegenden Band.

Golombok, Susan/Murray, Christopher/Jadva, Vasanti/MacCallum, Fiona/Lycett Emma (2004): Families created through surrogacy arrangements: parent-child relationships in the 1st year of life, in: Developmental Psychology 40/3, 400-411.

Golombok, Susan/MacCallum, Fiona/Murray, Christopher/Lycett, Emma,/Jadva, Vasanti (2006a): Surrogacy families: parental functioning, parent-child relationships and children' s psychological development at age 2, in: Journal of Child Psychology and Psychiatry 47/2, 213-222.

Golombok, Susan/Murray, Christopher/Jadva, Vasanti/Lycett, Emma/MacCallum, Fiona/Rust, John (2006b): Non-genetic and non-gestational parenting: consequences for parent-child relationships and the psychological wellbeing of mothers, fathers and children at age 3, in: Human Reproduction 21/7, 1918-1924.

Golombok, Susan/Readings, Jennifer/Blake, Lucy/Casey, Polly/Marks, Alex/Jadva, Vasanti (2011): Families created through surrogacy: mother-child relationships and children's psychological adjustment at age 7, in: Developmental Psychology 47/6, 1579-1588.

HFEA (2016): Fertility treatment 2014-2016 - Trends and Figures, März 2018, https://www.hfea.gov.uk/media/3188/hfea-fertility-trends-and-figures-2014-2016.pdf

HFEA (2017): Fertility treatment 2017 - Trends and Figures, Mai 2019, https://www.hfea.gov.uk/media/2894/fertility-treatment-2017-trends-and-figures-may-2019.pdf

ICMART (International Committee for Monitoring Assisted Reproductive Technologies), De Mouzon, Jaques/Chambers, Georgina M./Zegers-Hochschild, Fernando/Mansour, Ragaa/Ishihara, Osamu/Banker, Manish/Dyer, Silke/Kupka, Markus/Adamson, David G. 
(2020): World report: assisted reproductive technology 2012, in: Human Reproduction 35/8, 1900-1913.

Jadva, Vasanti/Murray, Christopher/Lycett, Emma/MacCallum, Fiona/Golombok, Susan (2003): Surrogacy: the experiences of surrogate mothers, in: Human Reproduction 18/10, 21962204.

Jadva, Vasanti/Blake, Lucy/Casey, Polly/Golombok, Susan (2012): Surrogacy families 10 years on: relationship with the surrogate, decisions over disclosure and children's understanding of their surrogacy origins, in: Human Reproduction 27/10, 3008-3014.

Kentenich, Heribert (2018): Leihmutterschaft: Überlegungen aus medizinischer und psychosomatischer Sicht, in: Gynäkologische Praxis 44/1: 47-52.

Koalitionsvertrag zwischen CDU, CSU und SPD. Deutschlands Zukunft gestalten, 18. Legislaturperiode, 16.12.20213, https://www.cdu.de/sites/default/files/media/ dokumente/koalitionsvertrag.pdf.

Leopoldina (Nationale Akademie der Wissenschaften) (2019): Stellungnahme: Fortpflanzungsmedizin in Deutschland - für eine zeitgemäße Gesetzgebung, März 2019, www.leopoldina.org.

Söderström-Anttila, Viveca/Wennerholm, Ulla-Britt/Loft, Anne et al. (2016): Surrogacy: outcomes for surrogate mothers, children and the resulting families - a systematic review, in: Human Reproduction Update 22/2, 260-276.

Tschudin, Sibil/Griesinger Georg (2012): Leihmutterschaft, in: Gynäkologische Endokrinologie 10, 135-138.

Van den Akker, Olga (2007): Psychological trait and state characteristics, social support and attitude to the surrogate pregnancy and baby, in: Human Reproduction 22/8, 2287-2295. 